\title{
Design and In Vitro/In Vivo Evaluation of Ultra-Thin Mucoadhesive Buccal Film Containing Fluticasone Propionate
}

\author{
Hussein O. Ammar, ${ }^{1,2,4}$ Mahmoud M. Ghorab, ${ }^{3}$ Azza A. Mahmoud, ${ }^{1,2}$ and Hend I. Shahin ${ }^{1}$
}

Received 13 November 2015; accepted 30 January 2016; published online 16 February 2016

\begin{abstract}
Fluticasone propionate is a synthetic corticosteroid drug distinguished by its potent antiinflammatory action with low systemic side effects in comparison to other corticosteroids making it a potential drug for local buccal delivery. The aim of the present study was to design mucoadhesive buccal film containing fluticasone that is aesthetically acceptable and could maintain local drug release for a sustained period to manage the sign and symptoms of severe erosive mouth lesions. Solvent casting technique was used in film preparation. Different polymeric blends were used either alone or in combination with mucoadhesive polymers, sodium carboxymethyl cellulose (SCMC), or Carbopol 971P at different concentrations. The physicochemical properties, in vitro mucoadhesion time as well as the drug release properties for all prepared formulations were determined. Selected formulations with adequate properties were further examined by differential scanning calorimetry (DSC) and X-ray diffraction (XRD) and subjected to in vivo evaluation. Films containing hydroxypropyl methylcellulose (HPMC)/ ethyl cellulose (EC) showed acceptable physicochemical properties, homogenous drug distribution, convenient mucoadhesion time, moderate swelling as well as sustained drug release up to $12 \mathrm{~h}$. The biological performance of these formulations was assessed on healthy human volunteers and compared with a prepared mouthwash which showed enhanced pharmacokinetic parameters for the selected films in comparison to the mouthwash. The results revealed that the optimized formulation containing HPMC/EC and $10 \%$ SCMC could successfully achieve sustained drug release for $10 \mathrm{~h}$ which is considered promising for local treatment of severe mouth lesions.
\end{abstract}

KEY WORDS: anti-inflammatory; buccal films; corticosteroids; fluticasone propionate; mouth lesions; mucoadhesive.

\section{INTRODUCTION}

For several years, the buccal route has served effectively in delivering many drugs that are susceptible to first-pass effect (1). It has many advantages such as ease of use, excellent accessibility, avoiding first-pass metabolism as well as enhancing patient compliance. In addition, it has been greatly used for local delivery of many drugs used in oral pathology. Consequently, the oral mucosa is characterized by attractive properties required for local and systemic drug delivery (2).

However, the low retention capability of dosage forms at the absorption site is considered one of the main disadvantages for the use of buccal route in drug delivery. The use of adhesive dosage forms has greatly improved drug delivery through the buccal cavity. The mucoadhesive drug delivery systems contain

\footnotetext{
${ }^{1}$ Department of Pharmaceutics and Pharmaceutical Technology, Faculty of Pharmaceutical Sciences and Pharmaceutical Industries, Future University in Egypt, Cairo, Egypt.

${ }^{2}$ Department of Pharmaceutical Technology, National Research Center, Dokki, Cairo, Egypt.

${ }^{3}$ Department of Pharmaceutics, Faculty of Pharmacy, Cairo University, Cairo, Egypt.

${ }^{4}$ To whom correspondence should be addressed. (e-mail: husseinammar@fue.edu.eg)
}

adhesive polymers which localize the dosage form on the buccal mucosa surface through the formation of secondary bonding (3).

One of the most used drugs in oral pathology is topical corticosteroids (TCs). They are commonly used for treating erosive lesions that affect the mucosa due to their potent antiinflammatory action (4). The contact time between the drug and the lesion is one of the main factors that plays crucial role in the success of the treatment with TCs. Consequently, mucoadhesive drug delivery systems such as adhesive films, tablets, and gels are considered effective in the treatment of oral diseases (5).

Generally, adhesive films have advantages over other mucoadhesive dosage forms as they are characterized by high flexibility and small size which enhance patient's comfort. Moreover, buccal films are specifically preferred in the case of local delivery for oral diseases as they offer the advantage of formation of a thin layer on the mouth lesion, thus protecting the wound surface and reducing the pain (6-8).

Fluticasone propionate (FP) is considered a potent corticosteroid drug that is used as anti-inflammatory in many diseases. Its optimized potency as well as topical activity along with low oral systemic potency and low mineralocorticoid activity, in comparison to other corticosteroids, make it a good candidate for treatment of diseases affecting the buccal mucosa such as severe erosive lesions affecting the oral mucosa (9). 
A study was carried out in order to examine the efficacy of topical FP spray and betamethasone sodium phosphate mouth rinse (BSP) in the treatment of mouth lesions. The study has revealed that both FP and BSP could effectively treat mouth lesions where a significant reduction in the surface area of lesions was observed. However, FP effectiveness was significantly higher than BSP $(p<0.05)(10)$. Although the results of this study were promising, however, no further studies were carried out in order to formulate fluticasone propionate in a suitable dosage form for the treatment of oral diseases.

The aim of the current study was to formulate mucoadhesive films containing low doses of fluticasone propionate, using different polymeric blends, for local treatment of severe erosive mouth lesions. This will ensure a satisfactory drug level in the mouth for prolonged duration of time. Incorporation of low drug dose will reduce possible side effects such as candidiasis. In addition, the local delivery of the drug will avoid side effects or drug interaction encountered during systemic therapy with corticosteroids. The formulation of elastic and smooth film requires the selection of suitable solvent and specific sequence for the addition of polymers and the poorly water-soluble drug which was investigated as a preliminary step in our study. The physicochemical and release properties of the prepared formulations were evaluated both in vitro and in vivo.

\section{MATERIALS AND METHODS}

\section{Materials}

Fluticasone propionate was kindly supplied by GlaxoSmithKline, Egypt. Hydroxypropyl methylcellulose (Methocel@ E4M premium; molecular weight (Mwt) $50,000 \mathrm{Da})$ and ethyl cellulose (Ethocel ${ }^{\circledR}$ std 10 premium; ethoxy content $49.5 \%$ ) were obtained from Colorcon, Italy. Polyvinylpyrrolidone (Mwt 13,000-23,000 Da) was obtained from Sigma-Aldrich, USA. Sodium alginate from brown algae (SALG) was purchased from Fluka, BioChemika, Switzerland. Chitosan $\mathrm{HCl}$ was obtained from Zhejiang Chemicals Import and Export Cooperation, China. Sodium carboxymethyl cellulose with high viscosity was supplied by Oxford Laboratory Reagents, India. Carbopol 971P NF (prop-2-enoic acid) were a gift from Lubrizol Advanced Materials, Italy. Propylene glycol was obtained from BDH Chemicals Ltd, England. Polyethylene glycol (Carbowax ${ }^{\mathrm{TM}}$ sentry TM 8000 powder NF; Mwt 70009000) was kindly supplied by Dow Chemical Company, India. Eudragit NE 30D (polyacrylate) and Eudragit RS 100 (ammonio methacrylate copolymer) were obtained from Rohm Pharma GmbH, Germany. Sodium hydroxide, ethanol 95\%, methanol high performance liquid chromatography (HPLC) grade, and acetonitrile HPLC grade were purchased from El Nasr Pharmaceutical Chemical Company, Egypt. Potassium dihydrogen phosphate $\left(\mathrm{KH}_{2} \mathrm{PO}_{4}\right)$ was obtained from ADWIC, Cairo, Egypt. Diflucortolone valerate (HPLC internal standard) was kindly supplied by Bayer Schering Pharma, Germany.

\section{Methods}

\section{Preparation of Fluticasone Propionate Mucoadhesive Films}

Fluticasone propionate mucoadhesive films were prepared using either a single polymer or different polymeric blends adopting solvent casting technique. However, initial screening with formulations containing single polymers showed unsatisfactory results. Consequently, single polymer formulations were excluded from our study. The binary mixture polymeric films (F1-F14) contained 2\% drug, 49\% plasticizer, and $49 \%$ film forming polymer. The amount of the film forming polymers was divided into (hydroxypropyl methylcellulose (HPMC)), ethyl cellulose (EC), or chitosan $\mathrm{HCl}$, with or without the addition of mucoadhesive polymer (sodium carboxymethyl cellulose or Carbopol). The total amount of the preparation was $0.5 \mathrm{~g}(w / w)$.

Propylene glycol (PG) was successfully used as a plasticizer in the investigated polymeric films except for chitosan $\mathrm{HCl}$ films, where polyethylene glycol (PEG) 8000 was incorporated as a plasticizer instead of PG due to incompatibility that occurred between the latter and chitosan $\mathrm{HCl}$.

Table I shows the composition of the prepared mucoadhesive films containing binary polymeric blends.

\section{Preparation of HPMC/EC Film With or Without Mucoadhesive Polymer}

The polymeric solution blend was prepared as follows: EC was accurately weighed and dissolved in $10 \mathrm{ml}$ ethanol, and HPMC was then added to the alcoholic solution. The polymeric solution was stirred for 30 min using magnetic stirrer and then sonicated to obtain a clear solution. PG was added to the polymeric solution slowly. Ten milligrams of the drug were dispersed in $5 \mathrm{ml}$ ethanol and then sonicated for $10 \mathrm{~min}$. Finally, the drug solution was added to the polymeric solution under continuous stirring. The film (F1) was prepared using solvent casting method, where the film forming solution was poured slowly into

Table I. Compositions of Fluticasone Propionate Polymeric Films

\begin{tabular}{|c|c|c|c|c|c|}
\hline \multirow{3}{*}{$\begin{array}{l}\text { Formula } \\
\text { code }^{a}\end{array}$} & \multicolumn{5}{|c|}{ Formula composition $(w / w \%)$} \\
\hline & \multicolumn{5}{|c|}{$\begin{array}{l}2 \% \text { drug and } 49 \% \text { plasticizer } \\
\text { with } 49 \% \text { polymers divided } \\
\text { into }\end{array}$} \\
\hline & HPMC & $\mathrm{EC}$ & $\begin{array}{c}\text { Chitosan } \\
\mathrm{HCl}\end{array}$ & SCMC & Carbopol \\
\hline $\mathrm{F} 1$ & 75 & 25 & - & - & - \\
\hline $\mathrm{F} 2$ & 70 & 25 & - & 5 & - \\
\hline F3 & 65 & 25 & - & 10 & - \\
\hline F4 & 55 & 25 & - & 20 & - \\
\hline F5 & 70 & 25 & - & - & 5 \\
\hline F6 & 65 & 25 & - & - & 10 \\
\hline F7 & 55 & 25 & - & - & 20 \\
\hline F8 & 75 & - & 25 & - & - \\
\hline F9 & 70 & - & 25 & 5 & - \\
\hline F10 & 65 & - & 25 & 10 & - \\
\hline F11 & 55 & - & 25 & 20 & - \\
\hline F12 & 70 & - & 25 & - & 5 \\
\hline F13 & 65 & - & 25 & - & 10 \\
\hline F14 & 55 & - & 25 & - & 20 \\
\hline
\end{tabular}

HPMC hydroxypropyl methylcellulose, $P V P$ polyvinyl pyrrolidone, $S A L G$ sodium alginate, EC ethyl cellulose, $S C M C$ sodium carboxymethyl cellulose

${ }^{a}$ Total film composition weight is $0.5 \mathrm{~g}$ 
plastic petri dish of $5 \mathrm{~cm}$ diameter. The casting solution was then dried at approximately $40^{\circ} \mathrm{C}$ for $24 \mathrm{~h}$. The dried film was cut into circles of diameter $0.5 \mathrm{~cm}$, packed in aluminum foil, and stored in glass containers at room temperature.

For the preparation of HPMC/EC medicated film containing mucoadhesive polymer, same previously mentioned steps were carried out to prepare polymeric solution containing the dissolved drug. After that, this solution was left to be evaporated at room temperature in order to obtain a solid dispersion. The calculated amount of sodium carboxymethyl cellulose or Carbopol $(5 \%, 10 \%$ and $20 \%)$ was dissolved in $15 \mathrm{ml}$ distilled water under continuous stirring, then it was mixed with the solid dispersion of the polymers and the drug. The polymeric dispersion was stirred continuously until a uniform preparation was obtained and was left overnight in the refrigerator to remove any entrapped air bubbles. The films (F2-F7) were prepared by solvent casting method as previously described.

\section{Preparation of HPMC/Chitosan HCl Films With or Without Mucoadhesive Polymer}

The polymeric solution was prepared by dissolving specified amount of HPMC in $15 \mathrm{ml}$ distilled water. Then, the polymeric solution was left to swell at room temperature. Chitosan $\mathrm{HCl}$ was added to the polymeric solution under continuous stirring. Specified concentration of PEG 8000 was first dissolved in $2 \mathrm{ml}$ ethanol, and then it was added to the polymeric solution. Ten milligrams of the drug was dispersed in $5 \mathrm{ml}$ ethanol and then sonicated for $10 \mathrm{~min}$. Finally, the drug solution was added to the polymeric solution under continuous stirring. The film (F8) was then prepared by solvent casting method as mentioned previously.

HPMC/chitosan medicated polymeric film containing mucoadhesive polymer was prepared as follows: calculated amount of sodium carboxymethyl cellulose or Carbopol $(5 \%, 10 \%$, and $20 \%)$ was dispersed in the least amount of distilled water, sonicated, and then added to a polymeric solution containing HPMC and chitosan $\mathrm{HCl}$. Ten milligrams of the drug was dispersed in $5 \mathrm{ml}$ ethanol and then sonicated for $10 \mathrm{~min}$. Specified concentration of PEG 8000 was first dissolved in ethanol and then was added to the drug solution. Finally, the drug solution was added to the polymeric solution under continuous stirring. The polymeric solution was stirred continuously until a uniform preparation was obtained and was left overnight to remove any entrapped air bubbles. The films (F9-F14) were prepared by the solvent casting method.

\section{Physicochemical Evaluation of the Prepared Polymeric Films}

\section{Film Thickness}

The thickness of the prepared films was measured using a micrometer (KM-211-101, Shaanxi, China). The thickness of each film at different three locations was measured, and the average thickness was determined (11).

\section{Determination of Drug Content Uniformity}

Spectrophotometric method was used to assess the uniformity of drug distribution through measuring drug content at different parts of the same film. Three circular cuts of each film $(0.5 \mathrm{~cm}$ in diameter) were weighed individually, dissolved in $10 \mathrm{ml}$ ethanol, and the solution was then filtered through $0.45 \mu \mathrm{m}$ Millipore ${ }^{\circledR}$ filter to remove the non-dissolved polymers and the concentration of fluticasone propionate was measured spectrophotometrically at $237.5 \mathrm{~nm}$ ( $\lambda_{\max }$ of the drug in ethanol). Each preparation was tested in triplicates (12), and the percentage drug content was calculated from the following equation :

$\%$ Drug content $=\frac{\text { Actual amount }}{\text { Theoretical amount }} \times 100$

\section{Determination of Weight Uniformity}

Three circular cuts of each film $(0.5 \mathrm{~cm}$ diameter $)$ were weighed individually. The average film weight values were determined (13).

\section{Determination of Surface $p H$}

The surface $\mathrm{pH}$ values of the films were measured in order to examine any possible side effects that could arise due to altered in vivo $\mathrm{pH}$. The film was moistened with $5 \mathrm{ml}$ of distilled water and then left to swell in a petri dish for $1 \mathrm{~h}$. The surface $\mathrm{pH}$ value was determined using $\mathrm{pH}$ meter (Jenway $\mathrm{pH} / \mathrm{mV} /$ Temperature Meter, model 3510, UK). The average of three measurements for each formulation was recorded (14).

\section{Folding Endurance}

The folding endurance is a measure of the film integrity and strength. The film was manually folded repeatedly at the same point until it broke or folded up to 300 times which is considered satisfactory to reveal good film properties (15).

\section{Determination of the Swelling Index}

The film (surface area, $1.75 \mathrm{~cm}^{2}$ ) was weighed and placed in a pre-weighed stainless steel wire sieve. The mesh containing the film sample was then placed in a petri dish containing $5 \mathrm{ml}$ phosphate buffer $\mathrm{pH} 6.8$ (16). At definite time intervals, the mesh was removed; absorbent tissue was used to remove excess water then the film was reweighed. Changes in film weight were determined at different time intervals. The degree of swelling was calculated using the formula (17):

S.I. $\%=\frac{W 2-W 1}{W 1} \times 100$

where S.I. is the swelling index, $W 2$ is the weight of film at time $t$ after swelling, and $W_{1}$ is the weight of the dry polymer film at zero time.

\section{In Vitro Drug Release Studies}

The release of fluticasone propionate from the prepared films was examined in phosphate buffer $\mathrm{pH} 6.8$ at $37 \pm 0.5^{\circ} \mathrm{C}$ 
(18). Each mucoadhesive film was adhered by cyanoacrylate glue to the beaker side wall (25 ml beaker) (19). Each covered vessel containing $10 \mathrm{ml}$ phosphate buffer $\mathrm{pH} 6.8$ was placed in a shaking incubator rotating at a rate of approximately 150 strokes per minute. After specific time intervals, $5 \mathrm{ml}$ sample was withdrawn, filtered through Millipore ${ }^{\circledR}$ filter of $0.45 \mu \mathrm{m}$ pore size, and assayed spectrophotometrically at $\lambda_{\max }$ of $235 \mathrm{~nm}$. To maintain the buffer volume in the vessel constant, each sample withdrawn from the release medium was compensated with similar volume of phosphate buffer $\mathrm{pH}$ 6.8. The absorbance of the polymeric additives was negligible and did not interfere with $\lambda_{\max }$ of the drug. Calculations were done on the basis of the calibration curve of the drug in phosphate buffer previously constructed. Drug release studies were done in triplicates. The cumulative percentage of drug released was plotted against time. The mechanism of the drug release from different mucoadhesive systems was determined by fitting the release data to different kinetic models.

\section{In Vitro Mucoadhesion Residence Time}

Fluticasone propionate mucoadhesive films with optimum release profile were selected for studying their in vitro mucoadhesion time. It was evaluated by recording the time required for these films to detach from a chicken pouch membrane in a well-stirred beaker filled with $500 \mathrm{ml}$ phosphate buffer $\mathrm{pH} 6.8$ at $37^{\circ} \mathrm{C}$. Cyanoacrylate glue was used to attach the chicken pouch membranes to the side wall of the beaker. A light force was applied to fix the film on the membrane. The beaker was placed on a magnetic stirrer at an approximate rate of $150 \mathrm{rpm}$ to simulate buccal movement (20).

\section{Differential Scanning Calorimetry (DSC) Studies}

Selected films with moderate swelling index, accepted drug release profile, and in vitro mucoadhesion time were evaluated by DSC thermogram analysis (DSC-50, Shimadzu, Kyoto, Japan) to determine the drug physical state in the prepared films as well as the physical mixtures. DSC studies were carried out on fluticasone propionate powder, the polymers (HPMC, EC, SCMC, Carbopol), casted medicated films, and its corresponding physical mixtures. The procedure involved heating an accurately weighed sample $(5 \mathrm{mg})$ encapsulated in an aluminum pan at a predetermined scanning rate $\left(10^{\circ} \mathrm{C} / \mathrm{min}\right)$ and over a predetermined temperature range from $30^{\circ} \mathrm{C}$ to $400^{\circ} \mathrm{C}$. Dry nitrogen gas was used as carrier gas with a flow rate of $25 \mathrm{ml} / \mathrm{min}$.

\section{Wide Angle X-ray Diffraction Studies (XRD)}

XRD pattern for pure ingredients and selected mucoadhesive medicated films as well as their corresponding physical mixture were recorded using X-ray diffractometer (Scintag Inc., USA) equipped with $\mathrm{CuK} \alpha$ as the source of radiation. The generator operating voltage and current were $45 \mathrm{kV}$ and $9 \mathrm{~mA}$, respectively. The scanning speed was $2 \% \mathrm{~min}$, and the $2 \theta$ scanning range was $5-60^{\circ}$. The $2 \theta$ values and the intensities of the peaks were compared for pure ingredients, the physical mixtures, and the casted films (21).

\section{Evaluation of In Vivo Bioadhesion Time and Patient Compliance of the Optimized Buccoadhesive Film}

The selected buccoadhesive films were evaluated in six healthy human volunteers aged 25-50 years. The research protocol (PI 486) was approved by the research ethics committee for experimental and clinical studies at the Faculty of Pharmacy, Cairo University. Each film was applied to the gingival mucosa above the canine tooth by pressing the film for $30 \mathrm{~s}$ (22). The following criteria were evaluated: (1) the strength of adhesion (very, moderately, slightly adhesive, or non-adhesive), (2) bitterness, (3) any signs of local irritation, and (4) the adhesion time, time for detachment of discs from the buccal mucus membrane.

\section{In Vivo Evaluation of Selected Fluticasone Propionate Mucoadhesive Films}

Based on the in vitro evaluation, two optimum mucoadhesive formulations containing $1 \mathrm{mg}$ fluticasone propionate were selected for further in vivo studies. Since there is no available commercial product for treatment of mouth ulcer containing fluticasone propionate, the formulations were compared to a prepared mouthwash solution containing the same drug dose dissolved in $10 \%$ ethanol and $30 \%$ propylene glycol, where the volume was completed with buffer solution $\mathrm{pH}$ 6.8. The mouthwash composition was selected according to preliminary study done in our laboratory (data not shown). Six adult healthy volunteers, three males and three females (age range 25-50 years), were enrolled in the study. The volunteers were randomly divided into three equal groups, each of two subjects. The study was performed using a simple randomized crossover design trial. On the morning of the experiment, blank saliva was withdrawn from each volunteer. Before $0.5 \mathrm{~h}$ of the experiment, food and water were inhibited. The volunteers were instructed to brush their teeth and place the film on the buccal mucosa between the cheek and gingival in the region of upper canine with slight pressure for $60 \mathrm{~s}$ or gargle with $5 \mathrm{ml}$ of the mouthwash for $5 \mathrm{~min}$. Saliva samples were collected by direct withdrawal from the sublingual area by a micropipette. Samples were obtained at 0.0, 0.5, 1.0, 1.5, 2.0, 2.5, 3.0, 3.5, 4.0, 5.0, 6.0, 8.0, and $10 \mathrm{~h}$ after drug administration. Samples containing saliva were centrifuged at $3500 \mathrm{rpm}$ for $20 \mathrm{~min}$. Then, the supernatant of the samples was separated and stored frozen until analysis. After a washout period of 1 week, the volunteers were crossed over to receive the other formulation. The same precautions were carefully considered. Saliva samples were analyzed for fluticasone propionate adopting a modified sensitive, selective, and accurate HPLC method, developed and validated before the study. Exactly, $0.75 \mu 1$ of internal standard solution containing $200 \mu \mathrm{g} / \mathrm{ml}$ diflucortolone valerate in methanol and $0.4 \mathrm{ml}$ acetonitrile were added to the saliva samples and then centrifuged for $10 \mathrm{~min}$ at $3500 \mathrm{rpm}$. The upper layer was then separated, filtered through $0.45 \mu \mathrm{m}$ Millipore ${ }^{\circledR}$ filter, and $20 \mu \mathrm{l}$ samples were injected into the HPLC column for analysis. The isocratic mobile phase consists of acetonitrile and $20 \mathrm{~mm}$ potassium dihydrogen phosphate buffer $\mathrm{pH} 6$ in a ratio of 70:30 $v / v$. The mobile phase was filtered and degassed. The flow rate was set to $1.5 \mathrm{ml} / \mathrm{min}$, and the column effluent was monitored continuously using UV detection at $254 \mathrm{~nm}$. Calculated 
concentrations of fluticasone in salivary were collected, and the following parameters were calculated for the formulated film and the prepared mouthwash: $C_{\max }(\mu \mathrm{g} / \mathrm{ml}), T_{\max }(\mathrm{h})$, area under the curve $(\mathrm{AUC})_{(0-10)}(\mu \mathrm{g} \mathrm{h} / \mathrm{ml}), \operatorname{AUC}_{(0-\infty)}(\mu \mathrm{g} \mathrm{h} /$ $\mathrm{ml})$, area under the first moment curve $(\mathrm{AUMC})_{(0-t)}\left(\mu \mathrm{g} \mathrm{h}{ }^{2} /\right.$ $\mathrm{ml}), \operatorname{AUMC}_{(0-\infty)}\left(\mu \mathrm{g} \mathrm{h}^{2} / \mathrm{ml}\right)$, and mean residence time (MRT) (h).

\section{RESULTS AND DISCUSSION}

\section{Physicochemical Characterization}

Table II summarizes the physical characteristics of the formulated mucoadhesive films. The thickness of all the formulae ranged between 0.18 and $0.32 \mathrm{~mm}$ with low standard deviation which indicates uniformity within each formulation. Determination of the drug content showed that the drug was homogeneously distributed throughout the film, where the coefficient of variation values was mostly below $10 \%$. This ensures that the casting solution properties and the preparation method were suitable to achieve homogenous drug dispersion throughout the casted films (23). The weight of the films ranged from 4.43 to $6.93 \mathrm{mg}$ with uniform weight distribution within each formulation. Most films were found to have $\mathrm{pH}$ values in the intended range (6.5-7.4). Hence, these films will not cause any irritation when applied to the mucus membrane. However, some formulations showed a slight deviation from the aforementioned range $(\mathrm{pH}>7.4)$. This might be due to the presence of some polymers that are mostly alkaline in nature, such as HPMC and SCMC (19). The folding endurance was determined by repeatedly folding one film at the same place till it broke or folded up to 300 times manually, which was considered satisfactory to reveal good film properties $(7,24)$. All films did not show any cracks even after folding for more than 300 times except for films containing chitosan $\mathrm{HCl}$ (F8-F14), where an obvious reduction in the folding endurance values was recorded. This indicates that chitosan $\mathrm{HCl}$ reduces the flexibility and mechanical strength of the medicated films, even in the presence of mucoadhesive polymers (SCMC or Carbopol).

\section{Swelling Index}

The swelling index of a mucoadhesive polymer is a crucial factor in controlling the adhesion property through the formation of weak bond (25). Water uptake causes the entangled polymer to relax thus exposing all the bioadhesive sites for bonding formation. The swelling rate of the polymer directly affects the rate of bond formation (26).

All the tested formulations showed gradual swelling during the tested period (data not presented).

Figure 1 shows the effect of incorporation of bioadhesive polymer on the swelling index of different mucoadhesive films, which presents the percentage of swelling index at $30 \mathrm{~min}$ (midpoint for the swelling index) for HPMC/EC and $\mathrm{HPMC} /$ chitosan $\mathrm{HCl}$ films in combination with $10 \%$ SCMC or $10 \%$ Carbopol. The concentration of $10 \%$ mucoadhesive polymer was specifically selected as it gave the highest swelling index for most polymers.

It was observed generally that the bioadhesive polymers enhance greatly the swelling capacity of all mucoadhesive films. There was no marked difference between the effects of adding Carbopol or SCMC on the swelling index of mucoadhesive films. Generally, SCMC is a water-soluble polymer that contains hydroxyl and carboxyl groups which enhance hydrophilicity of the polymers (27). Consequently, as the SCMC content increases in the polymeric blend, the hydrophilicity, as well as the rate of water sorption increases, this increases the swelling ratio in turn (28). Another probable explanation is that there is mutual repulsion that occurs between the negatively charged carboxylate groups of SCMC as the concentration increases in the blend matrix, which leads to polymer relaxation and an increase in the swelling index (29). This ultimately results in enhanced swelling of the system. On the other hand, Carbopol 971P is lightly cross-linked, thus opens up easily resulting in water uptake and great swelling (30).

\section{In Vitro Drug Release}

Figure $2 \mathrm{a}, \mathrm{b}$ shows the release profiles of fluticasone from different mucoadhesive films. The addition of SCMC at

Table II. Physicochemical Characterization of Mucoadhesive Films Containing Fluticasone Propionate

\begin{tabular}{|c|c|c|c|c|c|}
\hline Formula code & Thickness $^{a}(\mathrm{~mm})$ & Drug content $(\%)^{a}$ & Weight $(\mathrm{mg})^{a}$ & Surface $\mathrm{pH}^{a}$ & $\begin{array}{l}\text { In vitro mucoadhesion } \\
\text { time }(\mathrm{h})^{a}\end{array}$ \\
\hline $\mathrm{F} 1$ & $0.18 \pm 0.01$ & $93.64 \pm 0.0002$ & $5.43 \pm 0.49$ & $6.70 \pm 1.34$ & - \\
\hline $\mathrm{F} 2$ & $0.21 \pm 0.01$ & $108.23 \pm 0.0009$ & $5.50 \pm 0.26$ & $7.55 \pm 1.11$ & $9.75 \pm 0.35$ \\
\hline F3 & $0.22 \pm 0.01$ & $109.32 \pm 0.0001$ & $6.93 \pm 0.45$ & $7.02 \pm 1.25$ & $10.23 \pm 0.32$ \\
\hline F4 & $0.22 \pm 0.00$ & $114.25 \pm 0.0009$ & $5.57 \pm 0.49$ & $7.67 \pm 1.36$ & $7.40 \pm 0.14$ \\
\hline F5 & $0.19 \pm 0.01$ & $105.38 \pm 0.0010$ & $6.73 \pm 0.38$ & $7.62 \pm 1.27$ & $3.15 \pm 0.21$ \\
\hline F6 & $0.23 \pm 0.02$ & $90.04 \pm 0.0001$ & $6.87 \pm 0.55$ & $7.10 \pm 1.10$ & $8.05 \pm 0.07$ \\
\hline F7 & $0.23 \pm 0.01$ & $107.78 \pm 0.0006$ & $6.43 \pm 0.25$ & $7.52 \pm 1.13$ & - \\
\hline F8 & $0.32 \pm 0.03$ & $115.48 \pm 0.0005$ & $4.63 \pm 0.57$ & $6.61 \pm 0.11$ & $1.05 \pm 0.07$ \\
\hline F9 & $0.30 \pm 0.01$ & $91.45 \pm 0.0010$ & $5.07 \pm 0.51$ & $6.74 \pm 0.08$ & $12.00 \pm 0.00$ \\
\hline F10 & $0.21 \pm 0.02$ & $92.89 \pm 0.0004$ & $4.43 \pm 0.51$ & $6.41 \pm 0.37$ & $7.08 \pm 0.11$ \\
\hline F11 & $0.22 \pm 0.02$ & $99.16 \pm 0.0011$ & $6.13 \pm 0.55$ & $6.79 \pm 0.18$ & $3.25 \pm 0.35$ \\
\hline F12 & $0.32 \pm 0.02$ & $93.79 \pm 0.0003$ & $4.93 \pm 0.60$ & $6.65 \pm 0.04$ & $2.50 \pm 0.71$ \\
\hline F13 & $0.31 \pm 0.01$ & $90.43 \pm 0.0015$ & $6.13 \pm 0.71$ & $6.54 \pm 0.09$ & $3.08 \pm 0.11$ \\
\hline F14 & $0.32 \pm 0.02$ & $91.33 \pm 0.0014$ & $5.83 \pm 0.76$ & $6.60 \pm 0.08$ & $4.25 \pm 0.35$ \\
\hline
\end{tabular}

${ }^{a}$ Each value represents the mean $\pm \mathrm{SD}(n=3)$ 


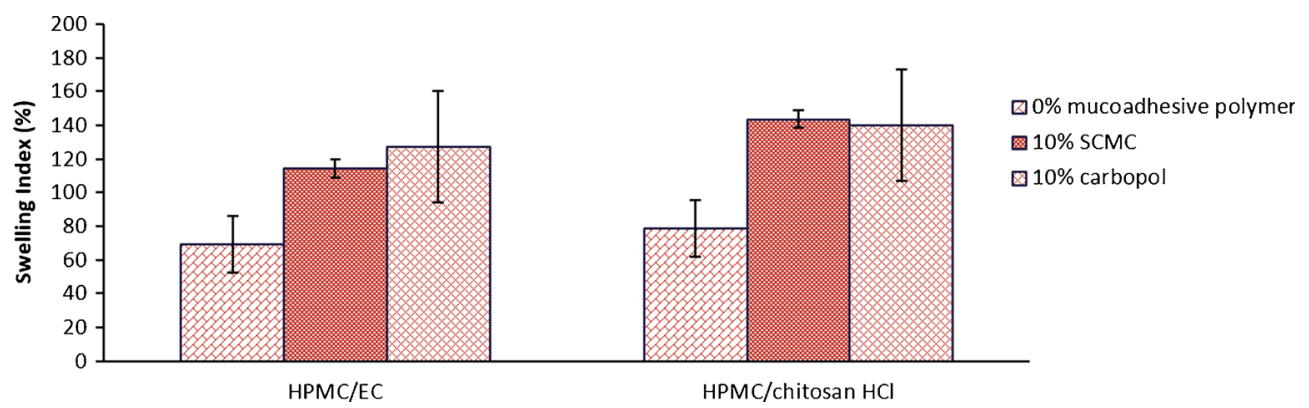

Fig. 1. The effect of addition of bioadhesive polymers on the swelling index of mucoadhesive films after $30 \mathrm{~min}$ of film insertion in $5 \mathrm{ml}$ phosphate buffer solution $\mathrm{pH} 6.8$

different concentrations to HPMC/EC (F2-F4) led to a decrease in the release efficiency of fluticasone propionate compared to HPMC/EC films without SCMC (F1). Hydrogen bond formation between the carboxyl group of SCMC and the hydroxyl group of HPMC could lead to an increase in cross-linking which in turn increased the viscosity and hence reduced drug release (19). In addition, the subsequent increase in the diffusional path length due to the high swelling index may be responsible for the low release profile (31). It was also observed that the addition of Carbopol (F5-F6) led to a decrease in the drug release efficiency significantly $(p<0.05)$ due to the formation of gelatinous layer upon hydration that protects the film against erosion. The release of drug from these systems may be delayed due to the interpolymer complexation network formed by the penetration of dissolution medium into the matrix as well as the presence of Carbopol that enables entrapment of the drug in the cross-linking structure $(26,32)$. However, further increase
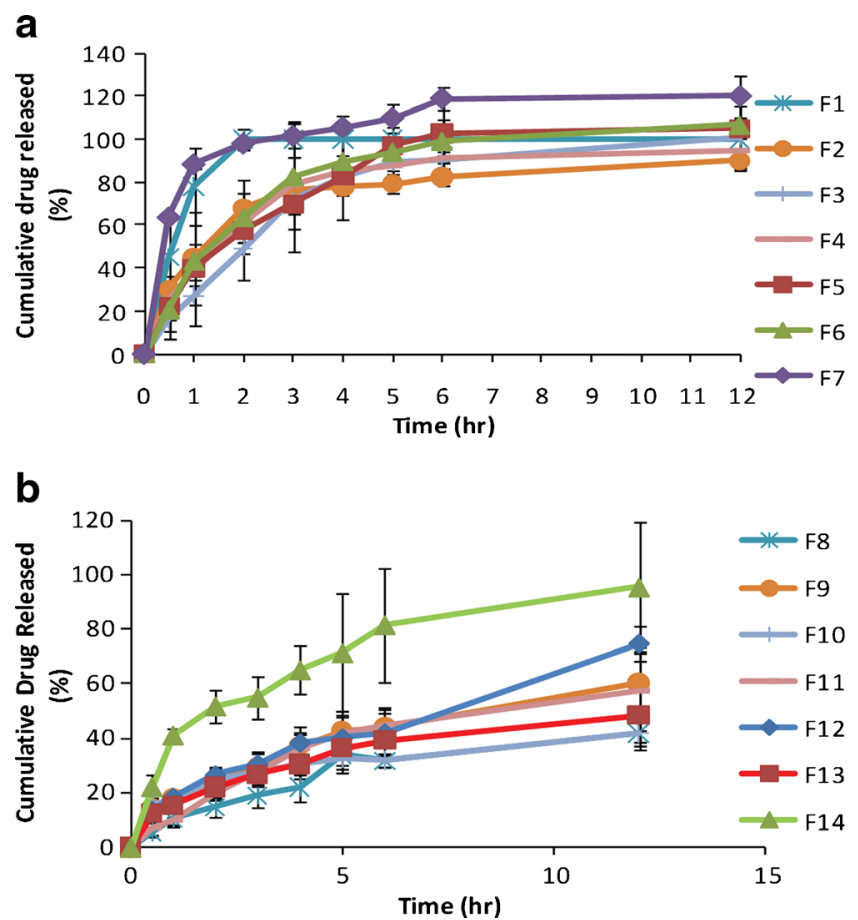

Fig. 2. a Release profiles of fluticasone propionate from mucoadhesive film containing hydroxypropyl methylcellulose/ethyl cellulose. b Release profiles of fluticasone propionate from mucoadhesive films containing hydroxypropyl methyl cellulose/chitosan $\mathrm{HCl}$ in Carbopol (F7) concentration led to an increase in the release efficiency.

It was found that the replacement of $\mathrm{EC}$ with chitosan $\mathrm{HCl}$ in film containing HPMC (F1 and F8, respectively) led to a significant decrease in the release of the drug $(p<0.05)$. This result is in agreement with the result reported by Mukherjee et al. (33) during the preparation of bilayered mucoadhesive delivery system for extended release of bisphosphonates. It was stated that interpolymer complexation occurred between HPMC and chitosan that led to controlled drug release. Another explanation for this result could be due to the possible interaction between hydroxyl group of fluticasone propionate and amino group of chitosan $\mathrm{HCl}$. Rodrigues and colleagues have reported an interaction between hydroxyl and amino group of chitosan and hydroxyl groups of dexamethasone (34). This interaction led to sustain drug release over $8 \mathrm{~h}$. In addition, chitosan films are characterized by slow erosion rate, which led to reduced drug diffusion through the polymer network (35).

The addition of SCMC led to non-significant change in the release efficiency $(p>0.05)$. On the contrary, an increase in the drug release efficiency was observed when $20 \%$ Carbopol was added in HPMC/chitosan $\mathrm{HCl}$ film (F14). This could be explained on the basis that the extent of interpolymer complexation depends on the ratio of HPMC to chitosan $\mathrm{HCl}$. Unlike (F12 and F13), HPMC concentration is considered relatively low in mucoadhesive film (F14). Consequently, this might affect the degree of interpolymer complexation. Therefore, the effect of Carbopol predominates over the interpolymer complexation effect in film (F14) which led to an increase in the amount of drug released. Bindu and Ravindra reported that at $\mathrm{pH} \mathrm{6.8,} \mathrm{Carbopol} \mathrm{is} \mathrm{present} \mathrm{in} \mathrm{its}$ ionized form which results in repulsion and polymer relaxation which leads to higher drug release (36).

The kinetic analysis of the in vitro drug release data of fluticasone from mucoadhesive films revealed that all formulae followed Higuchi model as a mechanism of drug release. Generally, according to Higuchi model, the drug molecules at the surface of the device are released, those in the center of the device having to migrate longer distances to be released which takes a longer time. This increased diffusion time results in a decrease in the release rate from the device with time. Generally, the rate of release is found to decrease in proportion to the square root of time (37). The release data were also fitted according to well-known Korsmeyer-Peppas diffusion model $(38,39)$ which is an exponential model to analyze drug release from polymeric devices with various geometrical shapes (40). As the Korsmeyer-Peppas diffusion model is valid only for early stages of release $(\leq 60 \%$ of the drug release) (41), this model 
cannot be applied on any formula with release profile more than $60 \%$ release in the first $1 \mathrm{~h}$. Most of the formulae were found to follow non-Fickian anomalous transport $(1>n>0.5)$, where the drug release is controlled by combination of diffusion and polymer relaxation, except for those containing HPMC/chitosan $\mathrm{HCl}$ with $5 \%$ and $10 \% \mathrm{SCMC}$ or $5 \%, 10 \%$, and $20 \%$ Carbopol (F9, F10, F12, F13, and F14, respectively), where the value of $n$ was less than 0.5 indicating Fickian transport where the rate of drug diffusion is much less than that of polymer relaxation (erosion) (data are not presented).

\section{In Vitro Mucoadhesion Time}

Table II shows the effect of bioadhesive polymers on the in vitro mucoadhesion time of different fluticasone films. It was observed that the mucoadhesive polymers (SCMC or Carbopol) increased the in vitro adhesion time of all mucoadhesive films. In general, SCMC containing films (F2, F3, F4, F9, F10, and F11) showed high bioadhesive strength values that were statistically significant $(p<0.05)$ than those for Carbopol containing films (F5, F6, F12, F13, and F14) with similar compositions. The carboxylic group of SCMC increases the mucoadhesive force through the formation of hydrogen bonds with the mucus membrane. In addition, the high swelling index of SCMC films encourages the polymer chain diffusion with the tissue thus increasing the adhesion force. All the aforementioned factors may have resulted in the superior bioadhesive force for the SCMC film (25). It was observed that films containing $10 \%$ SCMC achieve better mucoadhesion time than those containing $20 \%$ SCMC. On contrary, as the amount of Carbopol increases, the mucoadhesion time increases. This could be explained on the basis of the degree of erosion of the two polymers, where the increase in SCMC may result in increasing film porosity which facilitates dissolution medium penetration. This would consequently accelerate film erosion and detachment from the mucus membrane $(22,31)$. The mucoadhesion time ranged from 1 to $12 \mathrm{~h}$, according to the composition of the formulation.

From the above results, it was concluded that mucoadhesive films containing HPMC, EC, and 10\% SCMC polymers (F3) and film containing HPMC, EC, and 10\% Carbopol polymers (F6) are characterized by a sustained drug release profile with acceptable swelling rate and satisfactory in vitro mucoadhesion residence time. These formulas were thus selected for characterization by DSC and XRD and for investigation of their in vivo performance.

\section{DSC Studies}

The DSC was used in the present study to determine the physicochemical state of the drug in the HPMC/EC formulations containing 10\% SCMC or 10\% Carbopol (F3 and F6, respectively) and their corresponding physical mixtures.

Figure $3 \mathrm{a}$, b shows the thermogram of FP which represents one main characteristic endothermic peak at $277.87^{\circ} \mathrm{C}$, which corresponds to fluticasone propionate melting point. This result is in agreement with previous study carried out by Vatanara et al. (42) where fluticasone propionate exhibited endothermic behavior at $285-290^{\circ} \mathrm{C}$. The DSC thermograms of pure HPMC, EC, and SCMC are shown in Fig. 3a, which show a shallow, broad endothermic peak at $87^{\circ} \mathrm{C}, 184.4^{\circ} \mathrm{C}$, and $92.6^{\circ} \mathrm{C}$, respectively. Figure $3 \mathrm{~b}$ shows the same endothermic peaks for HPMC, EC in addition to a very shallow, broad endothermic peak for Carbopol that was observed at $245^{\circ} \mathrm{C}$. While a slight shifting in the endothermic peak of the drug was observed when mixed with the excipients in the physical mixture, where the characteristic endothermic peak of the drug appears at $313.47^{\circ} \mathrm{C}$ and $226.17^{\circ} \mathrm{C}$ instead of $277.87^{\circ} \mathrm{C}$ (Fig. 3a, b, respectively), which may be due to mixing of more than two substances, where the purity of each is reduced, leading generally to slight shifting in melting point reflected by the DSC endotherms (43). The disappearance of the endothermic peak corresponding to the melting point of FP in the HPMC/EC film (F3 and F6) indicated the dissolution of the drug crystals in the polymeric film (21).

\section{Wide Angle XRD Studies}

The wide angle XRD study was carried out to confirm the results of the DSC studies. The diffractograms of HPMC/EC formulations containing $10 \%$ SCMC or $10 \%$ Carbopol (F3 and F6) are illustrated in subpanels a and b of Fig. 4, respectively. The diffractograms of the pure drug and the pure films' components as well as their corresponding physical mixtures were added for comparison.

It was observed that the pure drug exhibited a diffraction pattern with numerous distinctive peaks indicating its highly crystalline state (44). The most abundant peaks were observed at $2 \theta$ values of $9.8^{\circ}, 13.0^{\circ}$, and $15.0^{\circ}$ and peaks of lower intensity $2 \theta$ values at $16.5^{\circ}, 18.0^{\circ}, 21.0^{\circ}$, and $22.0^{\circ}$.

The diffraction patterns of individual components (polymers) for HPMC/EC film (F3) are shown in Fig. 4a. HPMC diffraction pattern showed no characteristic peaks indicating its amorphous state. EC showed two broad peaks at $2 \theta=6.0^{\circ}$ and $11.0^{\circ}$. SCMC diffraction pattern showed two sharp peaks at $2 \theta=31.8^{\circ}$ and $45.0^{\circ}$, respectively. The diffraction patterns of individual components (polymers) for HPMC/EC film (F6) are illustrated in Fig. 4b which shows the same diffraction pattern previously mentioned for HPMC and EC as well as Carbopol diffraction pattern which showed no characteristic peaks indicating its presence in amorphous form. The XRD profile of the physical mixture for F3 also demonstrated the crystalline peaks for fluticasone propionate corresponding to $2 \theta$ values of $9.8^{\circ}, 13.0^{\circ}$, and $15.0^{\circ}$ as well as the two characteristic peaks for SCMC at $2 \theta=31.8^{\circ}$ and $45.0^{\circ}$. The physical mixture for F6 also showed the crystalline peaks for the fluticasone propionate. However, the peak intensity was lessened. This might be due to the relative decrease in the crystalline compound concentration in the physical mixture in comparison to the pure ingredients (45). The casted HPMC/ EC film containing 10\% SCMC (F3) showed one broad peak between $2 \theta=17.0^{\circ}$ and $25.0^{\circ}$, whereas the casted HPMC/EC film containing $10 \%$ Carbopol (F6) showed one broad peak between $2 \theta=16.0^{\circ}$ and $23.0^{\circ}$. This finding strongly suggests that the drug was distributed homogeneously in the polymeric matrix in amorphous state (21). This confirms the results obtained with the DSC study.

\section{Evaluation of In Vivo Bioadhesion and Patient Compliance of the Buccoadhesive Films}

In vivo testing of selected formulations containing $65 \%$ HPMC, $25 \%$ EC, and 10\% SCMC or 10\% Carbopol (F3 and 
a

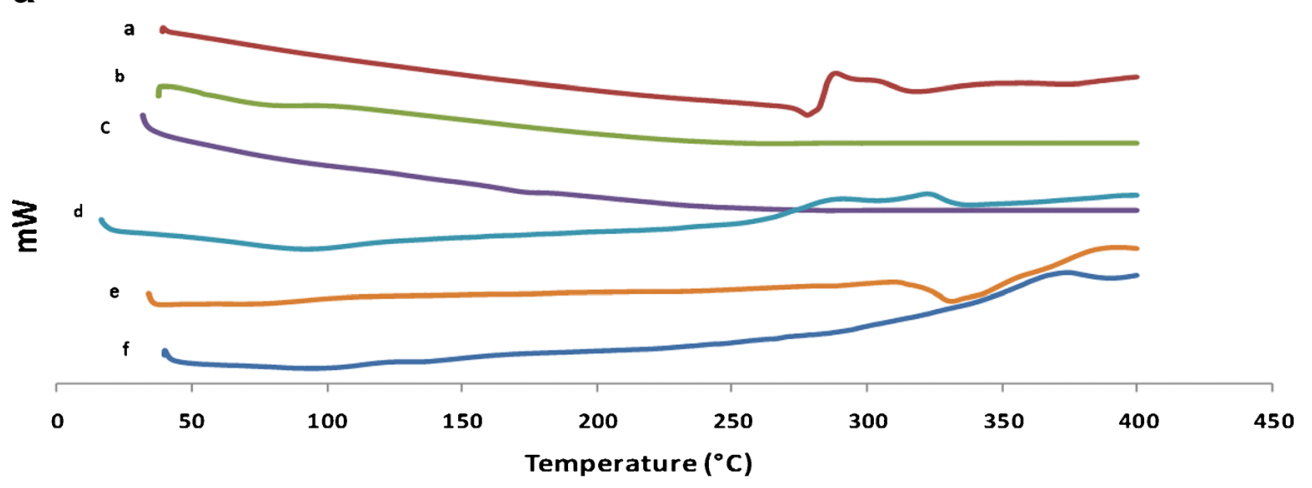

b

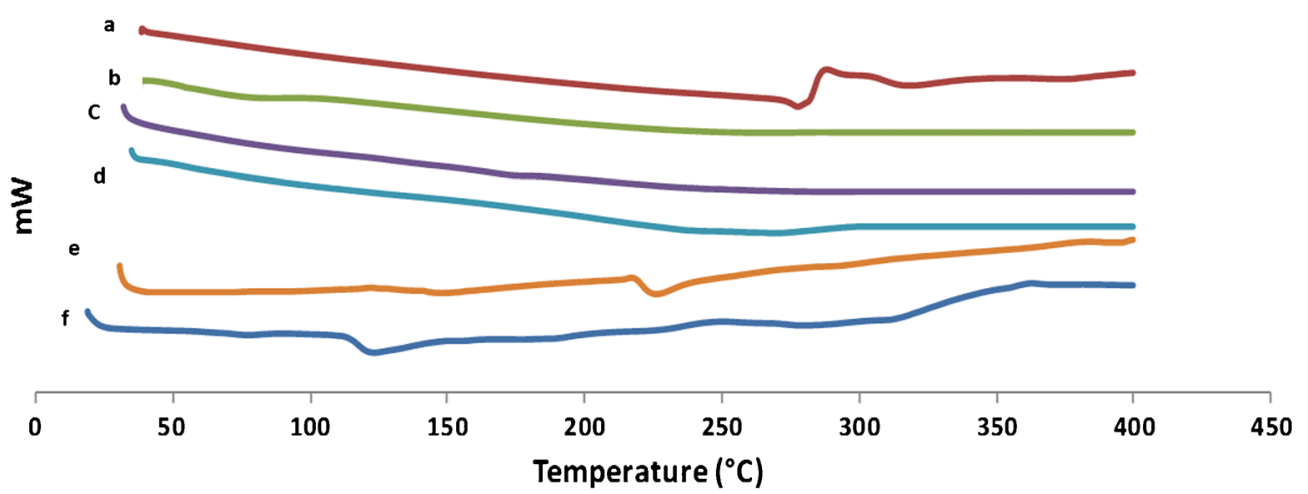

Fig. 3. a DSC thermograms of $a$ fluticasone propionate, $b$ hydroxypropyl methylcellulose, $c$ ethyl cellulose, $d$ sodium carboxymethyl cellulose, $e$ their corresponding physical mixture, and $f$ F3. b DSC thermograms of $a$ fluticasone propionate, $b$ hydroxypropyl methylcellulose, $c$ ethyl cellulose, $d$ Carbopol, $e$ their corresponding physical mixture, and $f \mathrm{~F} 6$

F6, respectively) on six healthy human volunteers showed that F3 can adhere to mucosa without detachment till $6 \mathrm{~h}$ while F6 can adhere till $4 \mathrm{~h}$, after which films began to detach from mucosa. This result is in agreement with in vitro mucoadhesion residence time study results indicating that the mechanism controlling the adhesion time is the same for in vivo or in vitro. However, the shorter in vivo mucoadhesion time is due to the buccal movement and salivary flow. Strength of adhesion was moderate (i.e., the volunteers did not complain from severe strength of adhesion nor looseness). No signs of irritation or feeling of bitterness until detachment of films from mucosa were observed nor reported.

\section{In Vivo Evaluation of Selected Fluticasone Propionate Mucoadhesive Films}

The in vivo evaluation of selected fluticasone propionate mucoadhesive films was carried out by measuring the salivary concentration of the drug over a period of $10 \mathrm{~h}$ using HPLC assay for either after gargling for 5 min with $5 \mathrm{ml}$ of mouthwash containing $20 \% \mathrm{w} / \mathrm{w}$ drug dissolved in phosphate buffer solution $\mathrm{pH} 6.8$ containing $10 \%$ ethanol and $30 \%$ propylene glycol or following the application of medicated mucoadhesive films to the gingival mucosa above the canine tooth containing the same dose of drug. The HPLC techniques were validated before the study and showed sufficient sensitivity, precision, and accuracy for detection of the salivary level of drug with the least complications.
The mean salivary drug concentration, illustrated in Fig. 5, demonstrates a striking difference in release rate between the mucoadhesive films and the mouthwash. Although high drug levels were observed in saliva following the use of mouthwash after the first $5 \mathrm{~min}$ of the experiment in comparison to lower drug levels obtained from the mucoadhesive films (F3 and F6) up to $30 \mathrm{~min}$, a remarkable fluticasone propionate concentration was released from the mucoadhesive films (F6 and F3) after 3 and $4 \mathrm{~h}$, respectively, compared to traces of the drug obtained from the mouthwash. On the other hand, the detectable drug concentration was present in saliva even after the complete erosion of the films up to 8 or $10 \mathrm{~h}$ for F6 and F3, respectively, which indicates sustained drug release from the investigated formula in comparison to the fast release of drug from the mouthwash.

The pharmacokinetic parameters observed for the three tested formulations are presented in Table III. The table compiled the mean value of $C_{\max }, T_{\max }, \mathrm{AUC}_{(0-t)}$, $\mathrm{AUC}_{(0-\infty)}, \mathrm{AUMC}_{(0-t)}, \mathrm{AUMC}_{(0-\infty)}$, and MRT for the six individuals under investigation. The relatively high standard deviation (SD) values obtained from the in vivo data can be explained by the difference in salivary flow from one subject to the other, which controls film hydration and detachment from the mucus membrane (46). The delayed $T_{\max }$ of the mucoadhesive films indicates that they could successfully achieve sustained drug release in comparison to mouthwash. The significant difference $(p<0.05)$ between AUC values of the two mucoadhesive films and the tested 


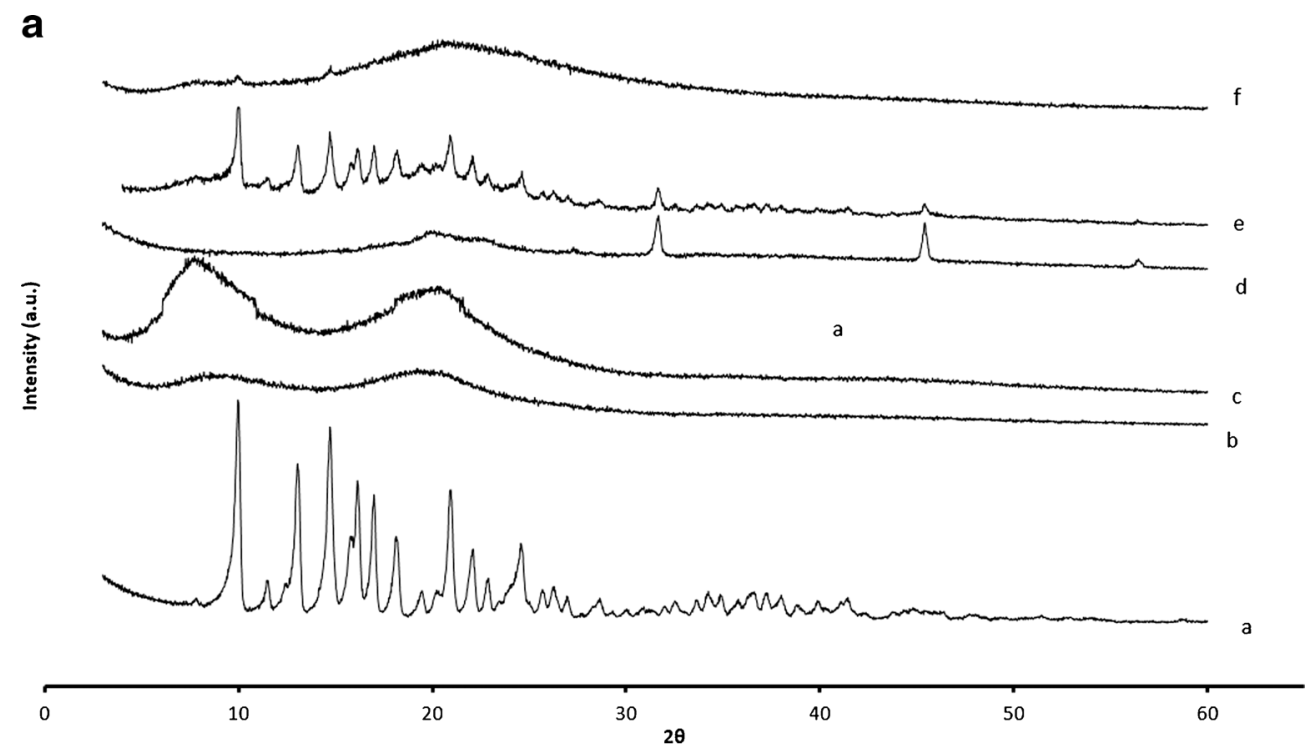

\section{b}

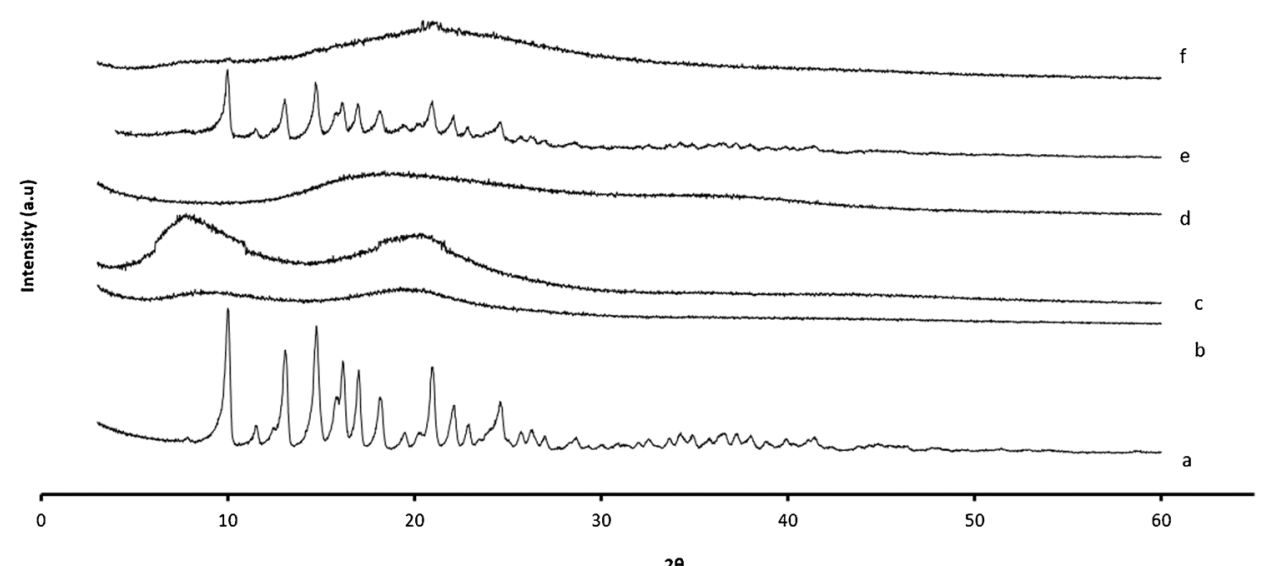

Fig. 4. a X-ray diffraction spectra of $a$ fluticasone propionate, $b$ hydroxypropyl methylcellulose, $c$ ethyl cellulose, $d$ sodium carboxymethyl cellulose, $e$ their corresponding physical mixture, and $f$ F3. b X-ray diffraction spectra of $a$ fluticasone propionate, $b$ hydroxypropyl methylcellulose, $c$ ethyl cellulose, $d$ Carbopol, $e$ their corresponding physical mixture, and $f \mathrm{~F} 6$

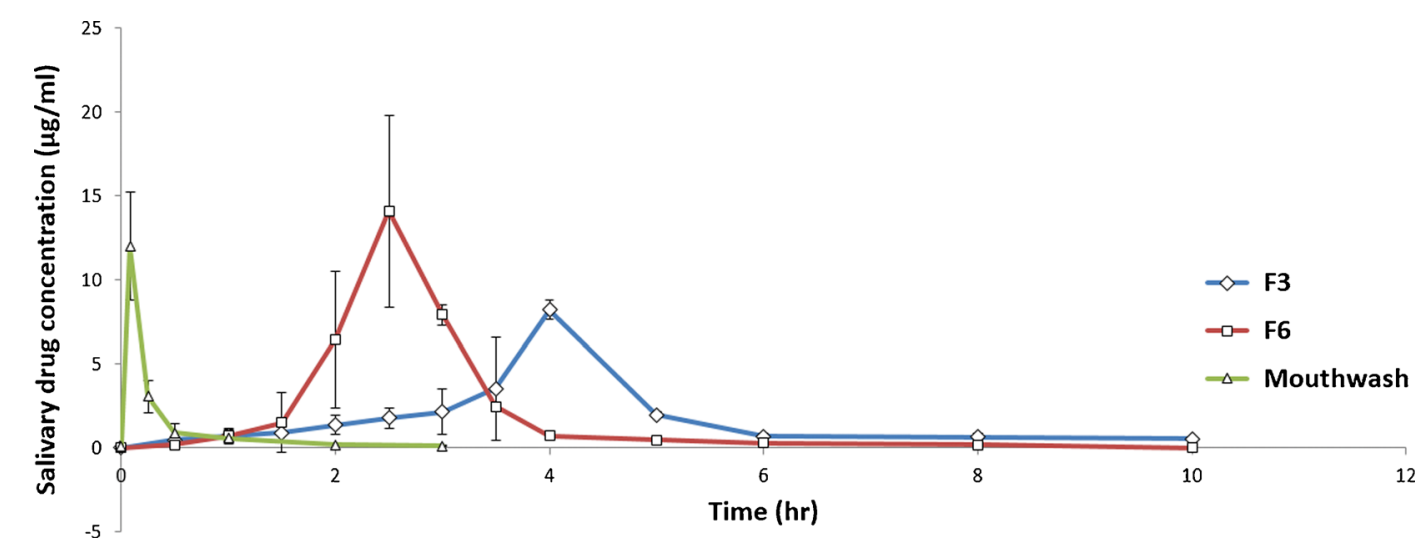

Fig. 5. Mean salivary concentrations of fluticasone propionate for six human volunteers after administration of mucoadhesive films (F3 and F6) and mouthwash 
Table III. Pharmacokinetic Parameters of Fluticasone Propionate Following the Application of a Single Dose of Mucoadhesive Films (F3 and F6) and Mouthwash

\begin{tabular}{|c|c|c|c|}
\hline \multirow[t]{2}{*}{ Pharmacokinetic parameter } & \multicolumn{3}{|l|}{ Tested formula } \\
\hline & F3 $($ Mean \pm SD $)$ & F6 $($ Mean \pm SD $)$ & $\begin{array}{l}\text { Mouthwash } \\
(\text { mean } \pm \text { SD) }\end{array}$ \\
\hline$C_{\max }(\mu \mathrm{g} / \mathrm{ml})$ & $8.33 \pm 2.97$ & $14.58 \pm 3.48$ & $8.83 \pm 3.04$ \\
\hline$T_{\max }(\mathrm{h})$ & $3.92 \pm 0.20$ & $2.65 \pm 0.38$ & $0.11 \pm 0.07$ \\
\hline $\operatorname{AUC}_{0-10}(\mu \mathrm{g} \mathrm{h} / \mathrm{ml})$ & $16.35 \pm 3.09$ & $18.29 \pm 3.58$ & $3.07 \pm 0.37$ \\
\hline $\mathrm{AUC}_{0-\infty}(\mu \mathrm{g} \mathrm{h} / \mathrm{ml})$ & $18.71 \pm 4.40$ & $18.83 \pm 3.47$ & $3.17 \pm 0.37$ \\
\hline $\operatorname{AUMC}_{0-10}\left(\mu \mathrm{g} \mathrm{h}^{2} / \mathrm{ml}\right)$ & $69.76 \pm 12.86$ & $49.39 \pm 9.07$ & $1.73 \pm 0.24$ \\
\hline $\operatorname{AUMC}_{0-\infty}\left(\mu \mathrm{g} \mathrm{h}^{2} / \mathrm{ml}\right)$ & $111.89 \pm 43.13$ & $55.48 \pm 7.64$ & $2.11 \pm 0.28$ \\
\hline MRT (h) & $5.27 \pm 1.38$ & $2.97 \pm 0.19$ & $0.64 \pm 0.09$ \\
\hline
\end{tabular}

$n=6$

$S D$ standard deviation

mouthwash indicates better availability of the drug from mucoadhesive films in comparison to mouthwash. The duration of action for the tested formulations which was determined by their MRT values showed significant difference $(p<0.05)$ between the three formulations which was the highest for film F3. This confirmed that the candidate film F3 was able to achieve sustained drug release for $10 \mathrm{~h}$.

It was previously estimated that $\mathrm{IC}_{50}$ (represents the concentration of fluticasone propionate required to produce $50 \%$ inhibition of eosinophil survival activities) for the anti-inflammatory effect of fluticasone propionate in human epithelial cells is $0.001 \mu \mathrm{g} / \mathrm{ml}$ (47). Fortunately, the results of our study revealed that the mucoadhesive film $\mathrm{F} 3$ in a dose equivalent to $1 \mathrm{mg}$ fluticasone propionate/ film was able to maintain the drug concentration in a range from 0.450 to $8.214 \mu \mathrm{g} / \mathrm{ml}$ for $10 \mathrm{~h}$ in the oral cavity. These results demonstrate that the prepared mucoadhesive film can attain reasonable fluticasone propionate concentration in the buccal cavity to treat painful oral lichens.

Consequently, the investigated mucoadhesive film containing $2 \%$ fluticasone propionate, $49 \%$ propylene glycol, and $49 \%$ polymer which is composed of $65 \%$ HPMC, $25 \% \mathrm{EC}$, and 10\% SCMC (F3) showed promising results and can be considered a suitable candidate that could successfully achieve sustained fluticasone propionate release, reduce dosing frequency, and maintain salivary drug concentration required for local treatment of severe mouth lesions for a prolonged time. It was also demonstrated that investigated film was ultra-thin, non-irritant, and tasteless, which is considered satisfactory for patient use.

\section{CONCLUSION}

The abovementioned data would, definitely, point out the suitability of formulating fluticasone propionate in the form of mucoadhesive buccal film containing low drug dose which reduces possible side effects. The suggested formulation could successfully achieve sustained drug release and maintain salivary drug concentration required for effective local treatment of severe mouth lesions which, consequently, will reduce dosing frequency and enhance patient compliance.

\section{COMPLIANCE WITH ETHICAL STANDARDS}

Conflict of Interest The authors declare that they have no competing interests. The authors alone are responsible for the content and writing of this article.

\section{REFERENCES}

1. Gibaldi M, Kanig JL. Absorption of drugs through the oral mucosa. J Oral Ther Pharmacol. 1965;1:440-50.

2. Mane PP, Bushetti SS, Keshavshetti GG. Development and invitro evaluation of mucoadhesive buccal films of nebivolol. Ind J Pharm Sci. 2014;76(2):166-9.

3. Valenta $C$. The use of mucoadhesive polymers in vaginal delivery. Adv Drug Deliv Rev. 2005;57(11):1692-712.

4. Venkatesh E, Bagewadi A, Keluskar V, A S. Role of corticosteroids in dentistry. Arch Dent Sci. 2010;1(1):3-11.

5. Gonzalez-Moles MA, Scully C. Vesiculo-erosive oral mucosal disease-management with topical corticosteroids: (2) protocols, monitoring of effects and adverse reactions, and the future. J Dent Res. 2005;84(4):302-8.

6. Donnelly RF, McCarron PA, Tunney MM, Woolfson AD. Potential of photodynamic therapy in treatment of fungal infections of the mouth. Design and characterisation of a mucoadhesive patch containing toluidine blue O. J Photochem Photobiol B. 2007;86(1):59-69.

7. Khanna R, Agarwal SP, Ahuja A. Preparation and evaluation of mucoadhesive buccal films of clotrimazole for oral Candida infections. Ind J Pharm Sci. 1997;59(6):299-305.

8. Singh S, Jain S, Muthu MS, Tiwari S, Tilak R. Preparation and evaluation of buccal bioadhesive films containing clotrimazole. AAPS PharmSciTech. 2008;9(2):660-7.

9. Crim C, Pierre LN, Daley-Yates PT. A review of the pharmacology and pharmacokinetics of inhaled fluticasone propionate and mometasone furoate. Clin Ther. 2001;23(9):1339-54.

10. Hegarty AM, Hodgson TA, Lewsey JD, Porter SR. Fluticasone propionate spray and betamethasone sodium phosphate mouthrinse: a randomized crossover study for the treatment of symptomatic oral lichen planus. J Am Acad Dermatol. 2002;47(2):271-9.

11. Kawarkhe S, Poddar SS. Designing of the mucoadhesive intravaginal spermicidal films. Indian J Pharm Sci. 2010;72(5):652-5. 
12. Poovi G, Umamaheswari M, Sharmila S, Kumar S, Rajalakshmi AN. Development of domperidone solid dispersion powders using sodium alginate as carrier. Euro J Appl Sci. 2013;5(2):3642.

13. Semalty A, Semalty M, Nautiyal U. Formulation and evaluation of mucoadhesive buccal films of enalapril maleate. Indian J Pharm Sci. 2010;72(5):571-5.

14. Mukherjee D, Bharath S. Design and characterization of double layered mucoadhesive system containing bisphosphonate derivative. ISRN Pharm. 2013;19:1-10.

15. Nafee NA, Boraie MA, Ismail FA, Mortada LM. Design and characterization of mucoadhesive buccal patches containing cetylpyridinium chloride. Acta Pharm. 2003;53(3):199-212.

16. Abdul Rasool B, Khan SA. In vitro evaluation of miconazole mucoadhesive buccal films. Int J Appl Pharm. 2010;2(4):23-6.

17. Parodia B, Russoa E, Cavigliolia G, Cafaggia S, Bignardia G. Development and characterization of a buccoadhesive dosage form of oxycodone hydrochloride. Drug Dev Ind Pharm. 1996;22:445-50.

18. Levy G. Effect of certain tablet formulation factors on dissolution rate of the active ingredient. J Pharm Sci. 1963;52(11):1039-46.

19. El-Samaligy MS, Yahia SA, Basalious EB. Formulation and evaluation of diclofenac sodium buccoadhesive discs. Int J Pharm. 2004;286(1-2):27-39.

20. Han RY, Fang JY, Sung KC, Hu OY. Mucoadhesive buccal disks for novel nalbuphine prodrug controlled delivery: effect of formulation variables on drug release and mucoadhesive performance. Int J Pharm. 1999;177(2):201-9.

21. Repka MA, Gutta K, Prodduturi S, Munjal M, Stodghill SP. Characterization of cellulosic hot-melt extruded films containing lidocaine. Eur J Pharm Biopharm. 2005;59(1):189-96.

22. Elfeky Y. Formulation and evaluation of anti-migraine bioadhesive buccal preparations. Faculty of Pharmacy, Cairo University, Egypt; 2011.

23. Woolfson A, McCafferty D, Moss G. Development and characterisation of a moisture-activated bioadhesive drug delivery system for percutaneous local anaesthesia. Int J Pharm. 1998;169(1):83-94.

24. Morales JO, McConville JT. Manufacture and characterization of mucoadhesive buccal films. Eur J Pharm Biopharm. 2011;77(2):187-99.

25. Peh KK, Wong CF. Polymeric films as vehicle for buccal delivery: swelling, mechanical, and bioadhesive properties. J Pharm Pharm Sci. 1999;2(2):53-61.

26. Anlar S, Capan Y, Hincal AA. Physico-chemical and bioadhesive properties of polyacrylic acid polymers. Pharmazie. 1993;48(4):285-7.

27. Nafee NA, Ismail FA, Boraie NA, Mortada LM. Mucoadhesive delivery systems. I. Evaluation of mucoadhesive polymers for buccal tablet formulation. Drug Dev Ind Pharm. 2004;30(9):985-93.

28. Taghizadeh M. Mechanochemical activation of carboxy methyl cellulose and its thermoplastic polyvinyl alcohol/starch biocomposites with enhanced physicochemical properties. Int J Biochem Biophys. 2013;1(1):9-15.

29. Taghizadeh MT, Sabouri N, Ghanbarzadeh B. Mechanochemical activation of carboxy methyl cellulose and Its thermoplastic polyvinyl alcohol/starch biocomposites with enhanced physicochemical properties. Int J Biol Biophys. 2013;1(1):9-15.
30. Sharmin N, Al-Mamun MD, Jalil R. A novel method to study the effect of $\mathrm{pH}$ and excipients on water uptake and swelling behaviour of Carbopol polymers. Bangl Pharm J. 2010;3(2):1-7.

31. Yehia SA, El-Gazayerly ON, Basalious EB. Fluconazole mucoadhesive buccal films: in vitro/in vivo performance. Curr Drug Deliv. 2009;6(1):17-27.

32. Ravikumar, Sachin RP, Patil MB, Paschapur MS, Mahalaxmi R. Formulation and evaluation of controlled-release diltiazem hydrochloride buccoadhesive tablets. Der Pharm Lett. 2010;2(1):48-60.

33. Mukherjee DJ, Bharath S, Madhavan V. Bilayered mucoadhesive delivery system for extended release of bisphosphonates. J Pharm Biol Sci. 2012;3(2):304-13.

34. Rodriguesa LB, Leitea HF, Yoshidab MI, Salibaa JB, Juniora AS, Faracoa AG. In vitro release and characterization of chitosan films as dexamethasone carrier. Int J Pharm. 2009;368(1-2):1-6.

35. Hassan MA, Barakat NS, El-Badry M, Shehata SM. Formulation and in vitro/in vivo evaluation of naproxen mucoadhesive buccal patches for local effect. J Drug Deliv Sci Tech. 2011;21(5):423-31.

36. Bindu RS, Ravindra RK. Formulation and in-vitro evaluation of mucoadhesive buccal patches pf omeprazole. Int J Adv Pharm. 2014;4(1):42-7.

37. Hillery AM. Advanced drug delivery and targeting: an introduction. In: Hillery AM, Lloyd AW, Swarbrick J, editors. Drug delivery and targeting for pharmacists and pharmaceutical scientists. 1st ed. New York: Taylor \& Francis Inc; 2001. p. 63-9.

38. Korsmeyer RW, Gurny R, Doelker E, Peppas NA. Mechanisms of solute release from porous hydrophilic polymers. Int J Pharm. 1983;15(1):25-35.

39. Ritger PL, Peppas NA. A simple equation for description of solute release II. Fickian and anomalous release from swellable devices. J Control Release. 1987;5(1):37-42.

40. Ozyazici M, Gokce EH, Ertan G. Release and diffusional modeling of metronidazole lipid matrices. Eur J Pharm Biopharm. 2006;63(3):331-9.

41. Desai KG, Kumar TM. Preparation and evaluation of a novel buccal adhesive system. AAPS PharmSciTech. 2004;5(3):1-9.

42. Vatanara A, Rouholamini NA, Gilani K, Asgharian R, Darabi M, Rafiee TM. Precipitation of fluticasone propionate microparticles using supercritical antisolvent. DARU J Pharm Sci. 2009;17(1):612.

43. Mura P, Manderioli A, Bramanti G, Furlanetto S, Pinzauti S. Utilization of differential scanning calorimetry as a screening technique to determine the compatibility of ketoprofen with excipients. Int J Pharm. 1995;119(1):71-9.

44. Llabot JM, Palma SD, Manzo RH, Allemandi DA. Design of novel antifungal mucoadhesive films. Part II. Formulation and in vitro biopharmaceutical evaluation. Int J Pharm. 2007;336(2):263-8.

45. Pattnaik S, Swain K, Mallick S, Lin Z. Effect of casting solvent on crystallinity of ondansetron in transdermal films. Int J Pharm. 2011;406(1-2):106-10.

46. Nafee NA, Ismail FA, Boraie NA, Mortada LM. Mucoadhesive buccal patches of miconazole nitrate: in vitro/in vivo performance and effect of ageing. Int J Pharm. 2003;264(1-2):1-14.

47. Ferrer JR, Mullol J, Lopez E, Xaubet A, Pujols L, Fernández JC, et al. Effect of topical anti-inflammatory drugs on epithelial cellinduced eosinophil survival and GM-CSF secretion. Eur Respir J. 1997;10(7):1489-95. 